\title{
Pre-medication for tracheal intubation
}

\author{
Amélia Gorete A. da Costa Reis*
}

$T$ laryngoscopy and tracheal intubation are generally shortlived, however, in certain circumstances, they may become more serious and long-lasting, and manifest as apnea, nausea, coughing, laryngospasm and bronchospasm, with consequent gastroesophageal reflux, difficulties viewing the larynx, obstruction of the airway, hypoxia, hypercapnia, pulmonary edema reduced cardiac output. In addition to respiratory reactions, direct laryngoscopy also alters cardiovascular function and provokes arterial hypertension, tachycardia or bradyarrhythmia and a significant increase in intracranial and intraoccular pressure. Rapid sequence intubation (RSI) consists of the appropriate use of medication to reduce the duration of direct laryngoscopy, facilitate the passage of the tracheal tube and reduce the adverse effects of this procedure. ${ }^{1}$ It is an organized approach that involves the use of analgesic, sedative and paralyzing agents. ${ }^{2}$ Rapid sequence intubation is a term applied in emergency care medicine and it implies control of the airway via tracheal intubation in a rapid and safe manner. ${ }^{1,2}$

Tracheal intubation prognosis should be measured, not just based on placement or not of the tube in the trachea, but also on reduction of the complications inherent in the procedure, i.e. adverse effects caused by direct laryngoscopy and by anesthetic induction. Rapid sequence intubation should be indicated, by a trained professional, after criteriabased evaluation of each patient, weighing the risks and benefits. ${ }^{3}$ Despite the possible complications, RSI has had its safety and efficacy documented as reducing the number of complications caused by tracheal intubation. $1,2,4,5$

\footnotetext{
* PhD, School of Medicine, Universidade de São Paulo. Assistant physician, Pronto-Socorro do Instituto da Criança, Hospital das Clínicas, School of Medicine, Universidade de São Paulo (USP).
}

Suggested citation: Reis AGAC. Pre-medication for tracheal intubation. J Pediatr (Rio J). 2004;80:341-3.
Rapid sequence intubation is considered routine procedure in emergency departments in the USA, being performed for 70 to $80 \%$ of tracheal intubations taking place at large academic centers. ${ }^{6}$ At hospitals that offer medical residency in pediatric emergency, records show a high rate of successful intubations using the RSI technique. $4,7,8$. It is the method of choice for the majority of emergency pediatric intubations since it is associated with a high success rate and low incidence of adverse reactions, when performed by a skilled professional, or when performed by Pediatrics residents under medical supervision. ${ }^{9}$

Rapid sequence intubation is indicated for patients requiring emergency tracheal intubation and, as they are rarely fasting, the risk of aspiration is considerable; ${ }^{4}$ as such when emergency intubation is performed, the necessary precautions against vomiting must always be taken.

Tracheal intubation with RSI is recognized as being faster and safer, even with children and infants and its benefits have been reported under a variety of situations. $4,10,11$ Nevertheless, the risks associated with the procedure are by no means negligible, with drug side effects and airway management difficulties being the greatest problems to be taken into account. The anticipation of and due care with the complications arising from a difficult airway or the adverse effects of the drugs employed is an integral part of RSI, ${ }^{12}$ which premise, together with the obligation to employ sequential steps, confers on RSI its value in emergency medicine.

It is extremely important to publish work describing the experience of Brazilian medical centers, as in the article by Bonow et al. published in this issue. ${ }^{13}$ This study reveals interesting data and certain features, already emphasized by the authors, deserve further comment.

It is odd that, even in intensive care units, in which there is an environment offering optimum conditions for emergency tracheal intubation, such as monitoring equipment and skilled professionals, both the administration of muscle relaxants to pediatric patients and of adequate sedation for newborns could be performed 
so infrequently. According to Bonow et al., sedatives were given to $89.5 \%$ of the pediatric patients and $24 \%$ of neonates, while muscle relaxants were used in just 3 and $0.9 \%$, respectively. This is probably the reality in the great majority of pediatric hospitals in Brazil, although training in intubation using RSI together with increased knowledge of the effects of sedatives, analgesics and muscle relaxants on children have a tendency to shift this panorama. The dissemination of studies such as this one by Bonow et al. demonstrates this tendency and greatly contributes to reflection on the subject of tracheal intubation. There is an obligation on the part of the professionals in the area to transform tracheal intubation into a less aggressive and invasive procedure for pediatric patients.

Bonow et al. report that prospective data collection was performed by means of interviewing the most senior doctor responsible for the intubation during the 48 hours immediately following the procedure; such conduct may have provoked some sort of bias since it is impossible to exclude the subjectivism thus involved. Recording data during the procedure would better reflect reality.

Bonow et al. describe that adequate relaxation was achieved in a small number of intubation attempts, in just $53.7 \%$ of the children and $31.9 \%$ of the newborn babies, this statistic could be worsened if the variables employed in the study are taken into account, i.e. questions on the presence of limb movements, jaw rigidity or coughing These parameters my not translate into the ideal degree of relaxation to permit laryngoscopy without resistance, an essential condition for reducing adverse effects. The present study corroborates this statement, since those patients that did present adequate muscle relaxation were intubated at the first attempt in just $59.7 \%$ of cases, with an even lower percentage being found for those without adequate muscle relaxation $(17.7 \%)$.

Difficulties during intubation were also more pronounced among patients with inadequate muscle relaxation ( $54.8 \mathrm{vs}$ $25 \% ; p=0.001$ ). While the definition of difficulties during intubation is unclear in this study, the low level of muscle relaxant employment, in this sample, together with the fact that these intubations were in general performed by medical residents undergoing training, explain the elevated percentage of "difficulties". A similar analysis can be made of the higher observed incidence of persistent hypoxemia among the patients with inadequate muscle relaxation (5.5 vs $23 \%$; $p=0.001$ ).

Bonow et al. observed at the PICU that patients with inadequate muscle relaxation received higher doses of sedatives ( 98.4 vs $81.9 \% ; p=0.004$ ), which conduct could result in a greater number of adverse events related to the sedatives. Sedatives and analgesics should be used to provoke analgesia and amnesia and reduce the level of consciousness, while in order to achieve adequate muscle relaxation neuromuscular relaxants must be used, which concept is the very basis of RSI. The authors offer in a most appropriate manner, during the discussion, the explanation for why doses above those recommended in literature are used, "This may be a result of the fact that the doses envisaged in the literature are recommended for RSI induction and not with the intention of achieving total muscle relaxation, in contrast with what, probably, happened with the patients in our study. Most probably, this muscle relaxation could have been achieved in a more appropriate manner by associating a short-duration muscle relaxant."

Intense sedation without paralysis cannot be considered to be a substitute for RSI. Performing intubation with a high dose of sedatives causes innumerable inconveniences, like procedure delay, loss of airway protection reflexes without the benefit of paralyzing them and even apnea. Some authors have demonstrated greater numbers tracheal intubation complications with intense sedation than with RSI. ${ }^{11}$

In the past, the inadequate use of neuromuscular relaxants lead to their being abandoned for emergency medicine; however RSI makes their use safe, since it implies step-by-step tracheal intubation, establishes a protocol and obliges professionals to foresee complications.

The authors explain, most adequately, that concern over the chance of being faced with a difficult airway contributes to the underemployment of neuromuscular relaxants, however, it must be pointed out that the first step in RSI is an adequate assessment of the airway whereas with intubation under sedation the chance of encountering a difficult airway is not generally contemplated, coming as a surprise.

The lack of time during emergency intubation cannot be accepted as justification for the lack of adequate medication, since the time spent preparing the patient prior to laryngoscopy is saved, reducing the period of hypoxemia. In the one situation in which time is truly essential and intubation must be as fast as possible, cardiorespiratory arrest, no medication whatsoever is indicated to facilitate the procedure.

The Pediatric Advanced Life Support course administrated by the Brazilian Society of Pediatrics (Sociedade Brasileira de Pediatria)in association with the National Resuscitation Council (Conselho Nacional de Ressuscitação) and the American Heart Association could contribute greatly to improving RSI skills, since, as Bonow et al. and other authors point out, ${ }^{14}$ there is a lack of specific, systematic, theoretical and practical training in this area.

\section{References}

1. Gerardi MJ, Sacchetti AD, Cantor RM, Santamaria JP, Gausche $M$, Lucid $W$, et al. Rapid-sequence intubation of pediatric patient. Ann Emerg Med. 1996;28:55-74.

2. Sakles JC, Laurin EG, Rantapaa AA, Panacek EA. Airway management in the emergency department: a one year study of 610 tracheal intubations. Ann Emerg Med. 1998;31:325-32.

3. Konrad C, Schupfer G, Wietlisbach M, Gerber H. Learning manual skills in anesthesiology: is there recommended number of cases for anesthetic procedures? Anesth Analg. 1998;86: 635-9.

4. Gnauck K, Lungo JB, Peter J, Nakanishi A. Emergency intubation of the pediatric medical patient: use of anesthetic agents in the emergency. Ann Emerg Med. 1994;23:1242-7. 
5. McAllister JD, Gnauck KA. Rapid sequence intubation of pediatric patient. Pediatr Clin North Am. 1999;46:1249-84.

6. Blanda M. Emergency airway management. Emerg Med Clin North Am. 2003;21:1-26.

7. Marvez-Valls E, Houry D, Ernst AA, Weiss SJ, Killeen J. Protocol for rapid sequence intubation in pediatric patients: a four-year study. Med Sci Monit. 2002;8:CR229-34.

8. Kramer DC, Grass G. Challenges facing the anesthesiologist in the emergency department. Cur Opinion Anaesthesiol. 2003; 16:409-16.

9. Sagarin MJ, Chiang V, Sakles JC, Barton ED, Wolfe RE, Vissers $\mathrm{RJ}$, et al. Rapid sequence intubation for pediatric emergency airway management. Pediatr Emerg Care. 2002;18:417-23.

10. Walls RM. Rapid-sequence intubation in head trauma. Ann Emerg Med. 1993;22:1008-13
11. Sing RF, Rotondo MF, Zonies DH, Schwab CW, Kauder DR, Ross $\mathrm{SE}$, et al. Rapid sequence induction for intubation by an aeromedical transport team: a critical analysis. Am J Emerg Med. 1998; 16:598-602.

12. Marvez E, Weiss SJ, Houry DE, Ernst AA. Predicting adverse outcomes in a diagnosis-Based Protocol System for Rapid Sequence Intubation. Am J Emerg Med. 2003;21:23-9.

13. Bonow FP, Piva JP, Garcia PC, Eckert GU. Avaliação do procedimento de intubação traqueal em unidades de referência de terapia intensiva pediátricas e neonatais. J Pediatr (Rio J). 2004;80:355-62.

14. Amantéa SL, Piva JP, Zanella MI, Bruno F, Garcia PCR. Acesso rápido à via aérea. J Pediatr (Rio J). 2003;79 Supl 2:S127-38.

\title{
The nutrition specialist's role in choosing special diets
}

\author{
Ary Lopes Cardoso*
}

$\mathrm{W}_{\mathrm{n}}$ in pediatric practice, a responsible medical posture is imperative. The study by Medeiros et al., ${ }^{1}$ published in this Jornal, deserves the readers' attention because it manages to demonstrate how this can and should be achieved.

Countless nutritional risks, the consequences of macro and micronutrient deficiencies, can be run when diets excluding certain foods are adopted. Several authors ${ }^{2-5}$ highlight a series of medium and long term problems, with the greatest emphasis, for patients under two years, accorded to diets excluding cow's milk.

Medeiros et al. demonstrate the nutritional deficits of the children studied by means of anthropometric data and by listing the intake of macro and micronutrients, especially of calcium. Irrespective of the results, the great merit of this work is its demonstration of the value of multidisciplinary involvement. The participation of the doctor, in conjunction with a nutritionist together with the social assessment are fundamental to guiding the choice of diet to that which most adequately substitutes the excluded items.

* PhD. Assistant physician; Chief of the Nutrology Unit, Instituto da Criança do Hospital das Clínicas, Faculdade de Medicina, Universidade de São Paulo (USP), Brazil.

Suggested citation: Cardoso AL. The nutrition specialist's role in choosing special diets. J Pediatr (Rio J). 2004;80:343-4.
Non-compliance with medical prescriptions has become a constant reality for Brazilian pediatricians. This is most notorious when the subject is exclusion diets. Nutritional risks are run, ${ }^{6}$ becoming more serious to the extent that they remain undetected ${ }^{7}$ or uncorrected. Some examples of risk situations, 8,9 and possibly even of immediate severity ${ }^{10}$ in which exclusion diets are imposed, have been described and discussed in the light of their nutritional aspects in work previously published in this Jornal.

Unfortunately, social, cultural and economic realities are the primary determinant factors in poor compliance with exclusion diet prescriptions. This is often translated into the diet being prepared inadequately, with insufficient (or excessive) nutrient intake; the allergic and/or inflammatory process being sustained by transgressions, allergy caused by substitute foods and varying degrees of malabsorption, both of macro and micronutrients, among others.

The participation of the nutrition specialist is necessary to the discussion and planning of care when an exclusion diet is being considered. Among other reasons are the capacity to assess nutritional requirements from anthropometric data and other body evaluation methods, to interpret the nutritional survey developed in conjunction with the nutritionist and for knowledge on the characteristics of the new ingredients that are contained in the new formulae and complete diets that are periodically launched onto the market in arithmetic progression. ${ }^{11,12}$ 